\title{
Codificación de mensajes: actividad de estudio e investigación utilizando praxeologías de álgebra lineal
}

\section{Message coding: study and research activity using linear algebra praxeologies}

\author{
Fermín Álvarez Macea ${ }^{\mathrm{a}}$, Viviana Angélica Costa ${ }^{\mathrm{b}}$, Cesar Augusto Hernández-Suárez ${ }^{\mathrm{c}}$ \\ ${ }^{a}$ Candidato a Doctor en Ciencias de la Educación, fermin.alvarez@udea.edu.co , https://orcid.org/0000-0002-2451-9144, Universidad de Antioquia, \\ Medellín, Colombia \\ bDoctora en Enseñanza de las Ciencias, vacosta@ing.unlp.edu.ar, https://orcid.org/0000-0003-1782-5378, Universidad Nacional de La plata, Argentina \\ 'Magister en Enseñanza de las Ciencias Básicas mención Matemática, cesaraugusto@ufps.edu.co, https://orcid.org/0000-0002-7974-5560, Universidad \\ Francisco de Paula Santander, Cúcuta, Colombia
}

Forma de citar: Álvarez Macea, F. R. ., Costa, V. A. ., \& Hernández-Suárez, C.A.. (2021). Codificación de mensajes: Actividad de estudio e investigación utilizando praxeologías de álgebra lineal. Eco

Matemático, 12 (2), 37-53

Recibido: 10 de Enero de 2021

Aceptado: 21 de Marzo de 2021

\section{Palabras clave}

Actividades de

estudio; instigación;

didáctica; álgebra lineal;

ingeniería; modelización

matemática; codificación

de mensajes;

metodología de

enseñanza

\footnotetext{
*Autor para correspondencia: cesaraugusto@ufps.edu.co
}

Resumen: Se presenta este artículo como parte de los resultados parciales del diseño e implementación de una Actividad de Estudio e Investigación, para la enseñanza de la codificación de mensajes, como un caso útil, con las Organizaciones Matemáticas del Álgebra Lineal en cursos de ingeniería. Anivel metodológico se adoptó una investigación cualitativa, de corte descriptivo e interpretativo, con elementos etnográficos, y método de observación participante. Se fundamenta en la Teoría Antropológica de lo Didáctico junto con sus constructos teóricos y didácticos. Se diseña un Modelo Praxeológico de Referencia, en el que se describen obras matemáticas relacionadas con el estudio de las praxeologías, la pregunta generatriz $\mathrm{Q} 0$ y sus respectivas preguntas derivadas $\mathrm{Q}_{\mathrm{i}}$ presentes en el desarrollo de la Actividad de Estudio e Investigación. Se implementa la Actividad de Estudio e Investigación con el surgimiento de varias formas de codificar y decodificar mensajes a la medida que se investiga y estudia para buscar posibles respuestas a la pregunta generatriz y sus derivadas. Con esto se evidencia una nueva forma en la enseñanza de Organizaciones Matemáticas del Álgebra lineal diferente a la que habitualmente se lleva a cabo en la educación superior en las facultades de ingeniería en Politécnico Jaime Isaza Cadavid. Finalmente, se establece que llevar este dispositivo didáctico al aula de clase permite minimizar en gran parte la monumentalización del saber, tal como se concibe dentro de la Teoría Antropológica de lo Didáctico 


\author{
Keywords \\ Study activities, \\ instigation; didactics; \\ Linear Algebra; \\ Engineering; \\ Mathematical Modeling; \\ Message Coding; \\ Teaching Methodology
}

\begin{abstract}
This article is presented as part of the partial results of the design and implementation of a Study and Research Activity, for the teaching of message coding, as a practical case, with Linear Algebra Mathematical Organizations in engineering courses. At the methodological level, a qualitative, descriptive, and interpretive research was adopted, with ethnographic elements, and participant observation method. It is based on the Anthropological Theory of the Didactic, together with its theoretical and didactic constructs, a Praxeological Reference Model was designed, in which mathematical works related to the study of praxeologies, the generating question Q0 and their respective derived questions Qi present were described. in the development of the Study and Research Activity. The Study and Research Activity was developed with the emergence of various ways to encode and decode messages as it was studied and investigated to find possible answers to the generating questions and their derivatives. With them, a new way of teaching Mathematical Organizations of linear Algebra was evidenced, different from what is usually carried out in higher education in engineering schools. Finally, it is evident that taking this didactic device to the classroom allows to minimize to a great extent the monumentalization of knowledge, as it is conceived within the Anthropological Theory of Didactics
\end{abstract}

\section{Introducción}

La enseñanza del Álgebra lineal es una de las asignaturas fundamentales en los programas de Ingenierías, por las relaciones y aplicaciones no solo con otras áreas, sino con situaciones problemas que se pueden abordar desde sus contenidos, aunque su nivel de abstracción y la descontextualización de situaciones problémicas reales, han sido un factor negativo a la hora de sus enseñanzas y por ende en su aprendizaje. El Álgebra Lineal permite explicar conceptos fundamentales y facilitar los cálculos en diferentes ingenierías, computación, economía, estadística, física y otras matemáticas, lo que se constituye en una herramienta esencial para un ingeniero (Álvarez-Macea, \& Costa, 2019).

Con la ayuda de la teoría Antropológica de lo Didáctico (TAD), se evidencia la perdida de sentido en la enseñanza del Álgebra Lineal, a lo cual Chevallard llama monumentalización de los saberes $(2004,2005,2007)$ o lo que Bernal (2019) denominan un estudio de un conjunto de obras muertas, para referirse a que el estudiante pasa por el aula de clase y no le encuentra sentido a lo que el sistema educativo escolar le ofrece. En este sentido, la enseñanza del Álgebra lineal en carreras de Ingeniería en grupos heterogéneos (estudiantes de diversos programas de ingeniería) carecen de situaciones de interés para los estudiantes, que estén cimentadas de forma transversal en cada formación disciplinar, como puede ser la codificación y descodificación de mensajes

Por otra parte, la implementación de laActividad de estudio e Investigación (AEI) como dispositivos didácticos dentro de la TAD, implica básicamente el estudio de cuestiones suficientemente ricas, vivas y fecundas que provoquen en los estudiantes la necesidad de seguir aprendiendo, y que facilite abrir un proceso de investigación, que permita explorar, conjeturar y validar. Por lo tanto, la codificación y descodificación de mensajes por medio de Organizaciones Matemáticas (OM) del Álgebra Lineal y su análisis a partir de las praxeologías planteadas en la TAD, por medio del dispositivo didáctico de las Actividad de estudio e Investigación en su diseño e implementación.

Es decir, las AEI dentro de la TAD, surgen como modelo didáctico que contrarresten el conocimiento acabado donde el docente no solo enseñe monumentos u obras muertas, sino por las praxeologías matemáticas que no surgen de manera instantánea en las instituciones ni aparecen de modo definitivo. Más bien por el contrario, son el 
resultado de un trabajo complejo y continuado que se realiza durante largo tiempo, en cuya dinámica de funcionamiento existen ciertas revoluciones invariables y que, por tanto, es posible modelizar (Bosch, García, Gascón \& Ruiz, 2006)

\section{Teoría Antropológica de lo Didáctico}

Los sustentos teóricos o el marco referencial, es la Teoría Antropológica de lo Didáctico (TAD), propuesta por Chevallard (1999) y desarrollada por otros investigadores durante los últimos años, que propone un modelo epistemológico para el estudio de la actividad humana en su dimensión institucional. Dos nociones son fundamentales en la TAD, la de institución y la de praxeología. La TAD ha desarrollado la noción de praxeología, un modelo para describir cualquier actividad humana regularmente realizada (Chevallard, 2001). En una praxeología, el saber se organiza en dos niveles: el de la praxis, ligado al saber-hacer y a los tipos de tareas, los problemas y las técnicas que se construyen y utilizan para abordarlos; y el del logos o saber, que se corresponde con los aspectos descriptivos, que organizan, por ejemplo, la actividad matemática.

La didáctica es la ciencia de lo didáctico o de los hechos didácticos, y que a ella le corresponde definir y redefinir este objeto (Chevallard, 2009). Lo didáctico se presenta en la multitud de situaciones sociales en las cuales alguna persona o alguna institución $\mathrm{Y}$, hacen algo -o pretenden intencionalmente hacerlo para que alguna persona $\mathrm{x}$, o $\mathrm{X}$ pueda estudiar una obra. Las obras son creaciones humanas materiales o no, producidas deliberadamente para cumplir una función definida (Chevallard, 2012). Lo didáctico supone la existencia de sistemas didácticos de tipo $\mathrm{S}(\mathrm{X}$; $\mathrm{y} ;)$, que funcionan según ciertas reglas, estudiadas por la Didáctica. Lo didáctico, también depende de las características de la obra, de la estructura de la disciplina a la que pertenece (temas y preguntas); así como de las estructuras de más alto nivel del campo praxeológico que integra (sectores y ámbitos). Sin embargo, aunque el análisis didáctico inicialmente consiste en el análisis praxeológico de la obra, en modo alguno se agota en esto (Chevallard, 2011)

\section{Actividades de Estudios y de Investigación}

Las Actividades de Estudios y de Investigación o AEI, propuestos por Chevallard (2004, 2007, 2009, 2007) son dispositivos didácticos que retoman la preocupación de la reconstrucción funcional de los saberes matemáticos, como respuesta a ciertas cuestiones fundamentales. Una AEI es, en principio, una organización didáctica donde la clase, bajo la dirección de un profesor, va a hacer estudiar, reconstruir y hacer accesible a los alumnos una cierta Organización Matemática Local (OML). Para esto es necesario partir de una cuestión generatriz Q cuyo estudio produzca la elaboración de una respuesta $\mathrm{R}$, y esta contenga los elementos esenciales de la OML inicial.

De esta manera, las AEI constituyen un proceso de estudio praxeológicamente finalizado, pues se imponela condición de que R contenga los principales componentes de una OML previamente determinada y conocida de antemano por la institución escolar. Una enseñanza por AEI permite comenzar a enfrentar el problema de la monumentalización de los saberes. Supone un cuestionamiento fuerte del contrato didáctico tradicional de la secundaria y cambios a nivel de mesogénesis, topogénesis y cronogénsis (Chevallard, 1985). Implica básicamente el estudio de cuestiones suficientemente ricas, vivas y fecundas que provoquen en los estudiantes la necesidad de seguir aprendiendo, y que facilite abrir un proceso de investigación, que permita explorar, conjeturar y validar.

Chevallard (1999), introdujo la distinción de diferentes tipos de OM, según el grado de complejidad de sus componentes: Organizaciones Puntuales (OMP): Están generadas por lo que se considera en la institución como un único tipo de tarea y está definida a partir del bloque práctico- 
técnico; Organizaciones Locales (OML): Es el resultado de integrar diversas praxeologías puntuales. Cada praxeología local se caracteriza por una tecnología que sirve para justificar, explicar, relacionar entre sí y producir las técnicas de todas las praxeologías puntuales que la integran; Organizaciones Regionales (OMR): Se obtienen mediante la coordinación, articulación y posterior integración de diversas praxeologías locales a una teoría matemática en común y Organizaciones Globales (OMG): Surgen al agregar varias praxeologías regionales a partir de la integración de diferentes teorías.

Por lo tanto, se elabora un Modelo Praxeológico de Referencia (MPR) relativo a la enseñanza de los temas que abarca cada AEI del Algebra Lineal, el cual se fue construyendo de manera natural con los estudiantes, identificando cada etapa (proceso) de la modelización, con los datos obtenidos en cada AEI o la utilización de un modelo ya diseñado, lo que permitió dar respuesta a las preguntas de investigación tales como: ¿Cuáles etapas del proceso de modelización están presentes en un curso cuando se implementa una AEI para el estudio con el Algebra Lineal en cursos de ingeniería?, ¿Cuáles praxeologías vinculadas al proceso de Modelización matemática, se construyen cuando se implementa una AEI en un curso de Algebra Lineal?. Así, dada la pregunta generatriz, se construye un diagrama con las posibles preguntas derivadas y las praxeologías matemáticas a estudiar para luego contrastar el MPR con la AEI desarrollada.

Lo anterior permite definir un proceso didáctico y metodológico para dar respuesta a la pregunta de investigación ¿Qué ventajas y desventajas surgen de la implementación de la pedagogía de la investigación y del cuestionamiento del mundo cuando se implementan AEI para el estudio con el Algebra Lineal en cursos de ingeniería?

\section{Método}

Se adoptó por una investigación cualitativa, de corte descriptivo e interpretativo, con elementos etnográficos, debido a la importancia de los aspectos sociales y además el contexto en el cual ocurren los acontecimientos (Hernández, Fernández \& Baptista, 2006), considerados de suma importancia dentro de la TAD (Chevallard, 2009). Como cualitativa y etnográfica, se opta por el método la observación participante (Rekalde, 2014), debido a que el docente investigador está inmerso en el escenario y en los acontecimientos que se llevan a cabo durante la investigación.

\section{Participantes y Escenario de la Investigación}

Los participantes hacen referencia a los estudiantes y el docente investigador, la observación se realiza a cursos habituales de Álgebra Lineal en los programas de Ingeniería de la institución de educación superior, de carácter público (oficial) Politécnico Jaime Isaza Cadavid (PJIC) en la ciudad de Medellín (Colombia), matriculados en el grupo (06) de la asignatura Algebra Lineal, la cual está ubicada en el segundo y tercer semestre, según el programa, de los planes de estudio.

Es importante resaltar, que el desarrollo de esta investigación se lleva a cabo en grupos heterogéneos en lo que concierne a los programas académicos que cursan los estudiantes. Así, en un mismo curso se pueden encontrar estudiantes de Ingeniería de Productividad y Calidad, Civil, Higiene y Salud ocupacional, Informática, Instrumentacióny Control. Además, un pequeño porcentaje de los estudiantes tienen formación previa (técnica o tecnológica) en áreas relacionadas con las ingenierías que cursan actualmente.

La población estudiantil del Politécnico Jaime Isaza Cadavid, tienen en promedio entre 35 y 40 estudiantes por grupo, con edades entre $18 \mathrm{y}$ 24 años, con un porcentaje de $52.84 \%$ de género masculino y $38.85 \%$ de género femenino, en la sede 
central, Poblado, provenientes de colegios públicos (en su mayoría) y privados de la ciudad de Medellín y su área metropolitana, de estrato socioeconómico 1,2 y 3 en su mayoría. Es pertinente mencionar, que los estudiantes de los cursos de Álgebra Lineal no tienen ninguna condición especial, para realizar lo concerniente a la intervención de las AEI y los procesos de Modelización.

Además, es importante mencionar que la investigación se llevó a cabo en el contexto indicado por la facilidad de acceso a la muestra, debido a la inmersión del investigador en el escenario educativo (Universidad) como docente en cursos de Álgebra Lineal en carreras de Ingeniería, con una experiencia de más de siete años, posibilitando el conocimiento acerca de los estudiantes en el sentido de su nivel académico, el contexto social educativo y la forma como se desarrolla el currículo de dicha asignatura.

El curso de Álgebra Lineal tiene una duración de 16 semanas (4 meses); semanalmente con dos secciones de clases, de dos horas cada una, para un total de 64 horas en el semestre. El contenido temático de la asignatura está conformado por 6 unidades temáticas; Álgebra de matrices y Sistemas de Ecuaciones Lineales, Determinantes, Vectores Geométricos y aplicaciones en R2 y R3, Espacios Vectoriales, Valores y Vectores propios, y Transformaciones Lineales.

Los escenarios pensados en la presente investigación son el aula de clase, los laboratorios, el aula-taller de la Universidad y en algunos casos, lugaresporfuera de la institución(empresasy espacios de la ciudad) donde el proceso de modelización matemática esté presente con temáticas del Algebra Lineal, en los cuales se desarrollen praxeologías que permitan la modelización de situaciones reales, que surjan de manera natural y espontánea.

\section{Análisis previo a la implementación de las AEI}

\section{Modelo Praxeológico de Referencia}

Para el estudio y análisis de praxeologías, es importante definir un Modelo Praxeológico de Referencia (MPR) y más, desde el lugar de evidenciar las diferentes etapas el proceso de modelización, es decir, el MPR se encuentra relacionado con lo que se entiende por enseñar y aprender matemáticas en una cierta institución. Profundizar en el análisis praxeológico de una obra, conduce a realizarse preguntas didácticas sobre la génesis institucional y sobre las transposiciones sucesivas que la han afectado (Corica y Otero, 2012), de esta manera las preguntas planteadas conllevan a tener un gran interés para determinar que transformaciones y evoluciones, se pueden hacer en el campo de la enseñanza, para modificar la enseñanza tradicional e implementar una nueva pedagogía de la investigación y cuestionamiento del mundo.

En relación con lo anterior, los constructos de la TAD plantean: La construcción y análisis de un MPR se encuadra en el nuevo paradigma de la pedagogía de la investigación y del cuestionamiento del mundo cuyo objetivo primordial es establecer una relación más funcional con el saber (Chevallard, 2012). En forma puntual y concreta se define la organización matemática local (OML), sobre la codificación o encriptación de mensajes, específicamente relacionadas con organizaciones matemáticas puntuales (OMP) del álgebra lineal que son de vital importancia para dar respuestas a la pregunta generatriz.

\section{Pregunta generatriz de la Actividad de Estudio e Investigación}

$Q_{0}$ : ¿Cómo se codifica o se encriptan mensajes? y sus respectivas preguntas complementarias o derivadas 
No obstante, para darle respuesta a dicha pregunta generatriz $\mathrm{Q}_{0}$ no se da de forma inmediata o directa, sino que implica el pensar otras cuestiones o características que se desprenden y surge la necesidad de ser estudiada a través de la construcción y/o de la OMP, en pro de hacer un análisis profundo, en lo que concierne a la respuesta.

En sintonía en la búsqueda de intentar responder $\mathrm{Q}_{0}$, se abre un abanico de diversas posibilidades que conllevan a la pregunta ¿Qué es codificar o encriptar? Al estudiar e investigar para dar respuesta a tal pregunta, resulta que existen formas de codificar o encriptar un mensaje y las partes que componen la codificación, tales como el emisor, el receptor, el sistema criptográfico o el convenio de un código definido entre quién emite el mensaje (emisor) y quien recibe el mensaje (receptor) y además el canal de comunicación. Así, de este modo podían generarse nuevos interrogantes o preguntas, tales como ¿Qué clase de código usar?, ¿Cómo se pueden organizar los datos del mensaje para codificarlo? En esta misma línea de construir la OMP para estudiar la codificación o encriptación, se encuentran las diferentes formas o etapas de generar la codificación, es decir, de hacerla manualmente o utilizar técnicas, tecnologías para determinar un modelo matemático que permita generalizar la generación de un proceso estándar en codificar y decodificar los mensajes, dando lugar al surgimiento de preguntas como ¿Qué clases o formas de codificación de mensajes existen?, ¿Cómo se genera la codificación y decodificación de mensajes?, ¿En el ámbito ingenieril cómo se usa la codificación?, ¿Qué tecnología o software se pueden utilizar para codificar mensajes?. Es importante resaltar que las preguntas y cuestiones no obedecen a un orden estricto, sino que van surgiendo en el proceso de estudio y de investigación de los estudiantes, relacionándolos siempre con las etapas del proceso de modelización matemática.

Por lo tanto, algunas relaciones o cuestiones que se pueden dan o surgir por la pregunta generatriz
$\mathrm{Q}_{0}$ son: $\mathrm{Q}_{0}$ ¿Cómo codificar o encriptar mensajes?; $\mathrm{Q}_{1}$ ¿Qué es codificar o encriptar?; $\mathrm{Q}_{2}$ ¿Qué clase de código es posible utilizar?; $\mathrm{Q}_{3}$ ¿Con cuáles datos o entradas se puede codificar?; $\mathrm{Q}_{4}$ ¿Qué codificación es posible realizar?; $\mathrm{Q}_{5}$ ¿Qué condiciones y características se debe cumplir para codificar mensajes?; $\mathrm{Q}_{6}$ ¿Cómo se genera la codificación y decodificación de mensajes?; $\mathrm{Q}_{7}$ ¿En el ámbito ingenieril cómo se usa la codificación?; $\mathrm{Q}_{8}$

¿Qué tecnología o software se utiliza para codificar los mensajes?

En correspondencia con la búsqueda de las respuestas a las preguntas anteriores, se pueden orientar la construcción o reconstrucción de las siguientes OMP del Álgebra Lineal ( Figura 1): OMP1: Organización de los datos en una matriz, OMP2: Orden de una matriz, OMP3: Operaciones con matrices, OMP4: Operaciones elementales sobre las filas (renglones) de una matriz, OMP5: Matriz escalonada y escalonada reducida OMP6: Determinante de una matriz, OMP7: Definición de matriz inversa, OMP8: Cálculo de la matriz inversa, OMP9: Sistema de ecuaciones lineales, OMP10: Reducción o eliminación gaussiana y Gauss-Jordán, OMP11: Naturaleza de la soluciones de un sistema lineal OPM12:Regla de Cramer OMP13: Vectores, operaciones y sus propiedades, OMP14: Conjunto y espacio generador, OMP15:Aplicaciones de la codificación y simulación. 


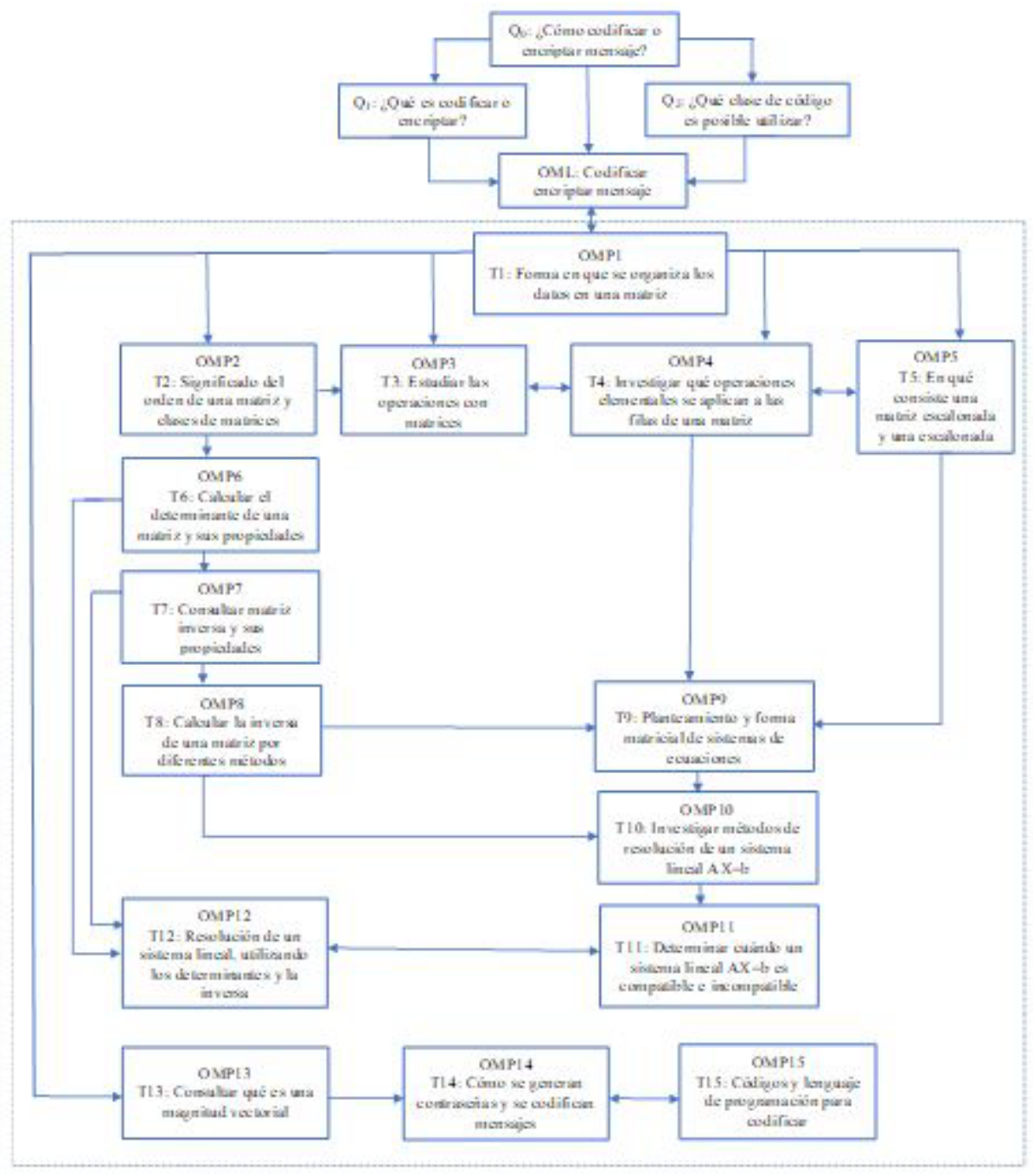

Figura 1. OMP del Álgebra Lineal

Fuente: Autor

El siguiente esquema muestra las cuestiones o preguntas derivadas de la pregunta generatriz $\mathrm{Q}_{0} \mathrm{y}$ las posibles OMP a construir o reconstruir en la medida de lo posible (Figura 2). 


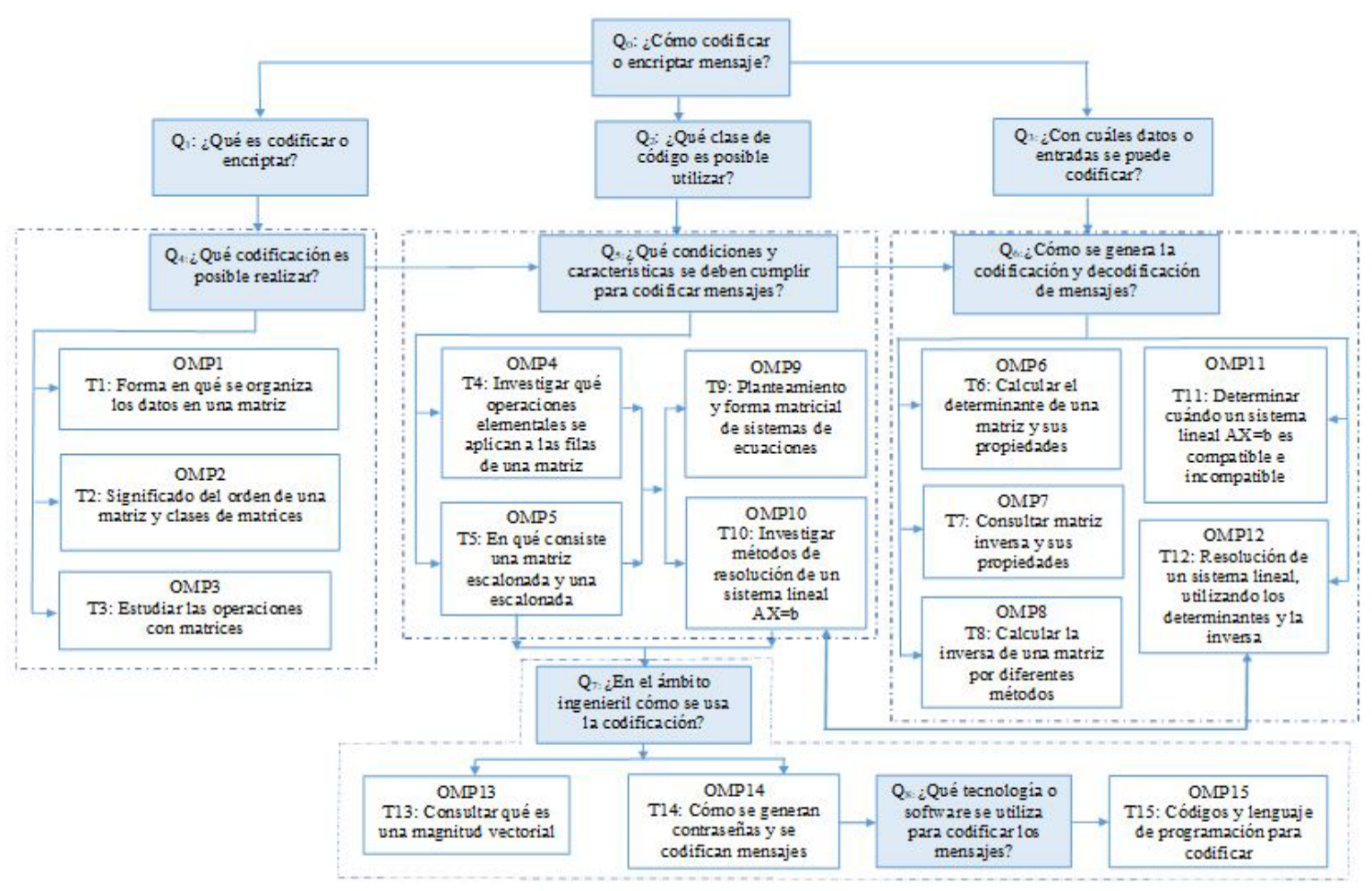

Figura 2. Esquema derivadas de la pregunta generatriz $\mathrm{Q}_{0}$ y las posibles OMP.

Fuente: Autor

\section{Implementación y descripción de las AEI}

La génesis de la cuestión o pregunta generatriz $\mathrm{Q}_{0}$ que se optó en el diseño de la Actividad de Estudio e Investigación, está relacionada con la temática de codificación o encriptación de mensajes, debido a su importancia en la actualidad y correlación con algunas organizaciones matemáticas del Álgebra Lineal.

En relación con el marco praxeológico de referencia (MPR), definido y construido anteriormente y en conjunto con la pregunta generatriz $\mathbf{Q}_{0}$ ¿Cómo se codifican o se encriptan mensajes?, a partir de esta cuestión inicial se desarrolló Actividad de Estudio e Investigación (AEI), donde para la búsqueda de dar respuesta a $\mathrm{Q}_{0}$ surgen preguntas derivadas a través de tipos tareas vinculadas a diferentes organizaciones matemáticas puntuales (OMP) del Álgebra lineal.
Las posibles respuestas van a estar dirigidas a la importancia de situaciones del contexto cotidiano real, donde está inmersa la codificación, cifrado o encriptación de mensajes, debido al desarrollo tecnológico en el cual hacemos parte a cada momento y en diferentes escenarios, como son las telecomunicaciones, los correos electrónicos, el almacenamiento de las bases de datos, el tratamiento de datos en redes sociales, seguridad informática, entre otros.

De acuerdo con lo anterior, despierta interés y un gran sentido en los estudiantes el estudio e investigación de actividades que ayuden a dar respuesta a la pregunta generatriz $\mathrm{Q}_{0}$ desde las praxeologías y la modelización matemática, lo que implica recorrer trayectorias en diversas organizaciones matemáticas $(\mathrm{OM})$ que se abordan desde el Álgebra Lineal, que van desde la parte 
conceptual sobre matrices, álgebra matricial, distintos sistemas de representaciones y varios desarrollos matemáticos, hasta llegar a comprender las aplicaciones de la codificación o encriptación de mensajes en múltiples contextos.

En el desarrollo de esta AEI se espera hipotéticamente permitir que el grupo de estudio en relación con los estudiantes participantes, cognitivamente desarrollen praxeologías (saberhacer) y las descripciones de las etapas de modelización, encuentren sentido a diferentes contenidos que se desarrollan en un curso de Álgebra Lineal en la facultad de ingeniería en el Politécnico Jaime Isaza Cadavid.

En esta organización didáctica (OD) para el desarrollo de esta AEI se implementó en el segundo semestre del año lectivo 2019 en el curso de Álgebra Lineal (CBS000408, grupo 6), durante las primeras 8 semanas del total del curso que son 16 semanas, para un total de 16 clases(sesión) de 2 horas cada una en el calendario académico, para el buen desarrollo de la AEI se tuvo presente las consideraciones contempladas en los niveles de codeterminación didáctica, en lo concerniente a nivel de Escuela (Universidad), Nivel de pedagogía-didáctica y nivel de disciplina-Área, en relación con los contenidos temáticos, el grupo docente, las horas de clases, el tiempo empleado y la forma de evaluación, para delimitar el desarrollo de la AEI, de igual forma los estudiantes se organizaron libremente en 11 grupos de trabajos y estudio, donde no todos quedaron del mismo número de estudiantes, cada grupo se reunía en el mismo espacio de trabajo, es decir, se agrupaban en forma circular con sus respectivas sillas, los cuales fueron conformados según lo establecido en los compromisos y acuerdos en el aula para el desarrollo de las AEI.

A manera, de descripción de las diferentes clases y la participación de cada uno de los grupos de estudio, se presenta la codificación de mensajes por medio de las praxeologías y OM del Álgebra lineal el desarrollo de AEI, dando las posibles respuestas a la pregunta generatriz $Q_{0}$ y las preguntas derivadas $\mathrm{Q}_{\mathrm{i}}$ :

¿Qué es codificar o encriptar?, donde participan los estudiantes mencionando y definiendo desde diferentes concepciones:

La codificación y encriptación, es unmecanismo, procedimiento de algoritmo criptográfico, donde se le debe asignar un valor numérico a cada carácter y definir un código entre las partes que se van a comunicar.

Que codificar es un proceso antiguo de diferentes culturas que consiste en ocultar un mensaje, ejemplo de ello es la cultura de la antigua Grecia donde los ejércitos de guerra como los espartanos utilizaban la transposición del mensaje que consistía en un esquema preestablecido para intercambiar los caracteres del mensaje original y utilizaban una clave simétrica.

¿Qué clase de códigos es posible utilizar? y ¿Con cuáles datos o entradas se puede codificar?

Los estudiantes por medio de sus computadores, smartphone y la búsqueda en internet, los textos guías de forma física y digital del curso de Álgebra lineal, determinan que pueden ser código de jerarquización, monocódigos, códigos numéricos o alfanuméricos, Código RSA, códigos de ASCII, código de Hufman, código Hill, código Morse, código de Hamming binario, los cuales se pueden organizar en una matriz. 

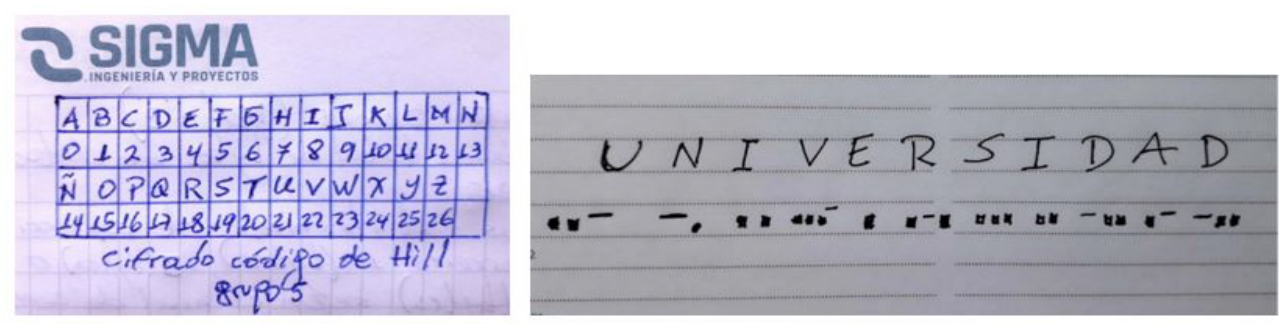

Figura 1. Códigos a utilizar

Fuente: Autor

¿Qué codificación es posible realizar? y a partir de esta pregunta se van estudiando las organizaciones matemáticas puntuales (OMP) del Álgebra lineal, que ayude a dar respuesta a la pregunta generatriz, a medida que los grupos de estudio van interviniendo con las posibles respuestas.

Se propone, estudiar la codificación de mensajes a partir de la utilización de matrices sin limitar las posibles codificaciones que se pueden realizar con otros contenidos, de modo que los estudiantes empiecen a estructurar como se organizan los datos en una matriz (OMP1) y provoque la búsqueda de diferentes aspectos del algebra matricial para codificar mensajes, tales como el orden de una matriz(OMP2) y las condiciones que se deben de dar para el procedimiento matemático, lo cual implica estudiar organizaciones matemáticas puntuales como las operaciones con matrices (OMP3), lo cual conlleva a realizar todas las tareas relacionadas con el equipamiento praxeológico, en relación con las operaciones elementales sobre las filas (renglones) de una matriz (OMP4), las clases de matrices y la definiciones de matriz escalonada y escalonada reducida (OMP5).

\section{Codificación de Mensajes con matrices.}

Para realizar codificación de mensajes con matrices, es importante determinar un código entre el emisor y el receptor del mensaje, para que de esta forma definir qué datos o entradas se van a utilizar para formar las matrices. Además, mencionan que se debe definir una matriz llave con ciertas características especiales y utilizar técnicas propias del Álgebra matricial, como es el producto de matrices y el cálculo de la matriz inversa.

\section{Paso para codificar o encriptar el mensaje (Figura X).}

1. Proceder a la asignación del número de cada letra (carácter), según el código a utilizar y formar la matriz mensaje por filas donde las entradas $\mathrm{a}_{\mathrm{ij}}$ son los números relacionados con los caracteres.

Tabla I. Código Definido

\begin{tabular}{|l|l|l|l|l|l|l|l|l|l|l|l|l|l|l|l|}
\hline A & B & C & D & E & F & G & H & I & J & K & L & M & N & N & O \\
\hline l & 2 & 3 & 4 & 5 & 6 & 7 & 8 & 9 & 10 & 11 & 12 & 13 & 14 & 15 & 16 \\
\hline P & Q & R & S & T & U & V & W & X & Y & Z & *, ESPACIO & & & & \\
\hline 17 & 18 & 19 & 20 & 21 & 22 & 23 & 24 & 25 & 26 & 27 & 0 & & & & \\
\hline
\end{tabular}

Fuente: Autor

2. Agrupar las letras del mensaje de a dos letras (caracteres), de tres, de cuatro o del número de agrupación que desee y así definir la matriz llave $\mathrm{A}_{\mathrm{nxn}}$. 
3. Realizar el producto de la matriz mensaje por la matriz llave $\mathrm{A}_{\mathrm{nxn}}$ y se obtiene la matriz codificada.

Pasos para descodificar o desencriptar un mensaje. (Figura 4)

1. Calcular el determinante de la matriz llave $\mathrm{A}_{\mathrm{nxn}}$, para comprobar que es una matriz no singular (invertible), además explican para hacer énfasis que una de las condiciones es que el determinante debe ser distinto de cero $(\operatorname{det}(A) \neq 0)$.

2. Se calcula la matriz inversa $\left(\mathrm{A}^{-1}\right)$ de la matriz llave $\mathrm{A}_{\mathrm{nxn}}$, utilizando cualquiera de los procedimientos vistos anteriormente.

3. Se procede a realizar el producto de la matriz codificada por matriz inversa $\left(\mathrm{A}^{-1}\right)$ y se obtiene la matriz mensaje.

4. Se sustituye cada número por el valor de la letra (carácter) del código definido, teniendo en cuenta las entradas por cada fila de la matriz mensaje.

Ejemplo propuesto: El siguiente mensaje de Facebook está codificado en forma matricial,

$$
\left[\begin{array}{ccc}
-10 & 74 & 104 \\
-5 & 6 & 11 \\
-2 & 64 & 87 \\
16 & -7 & -4
\end{array}\right] \text { decodificar y escribir dicho }
$$
mensaje, conociendo la matriz llave $\left[\begin{array}{ccc}0 & 3 & 4 \\ 1 & -4 & -5 \\ -1 & 3 & 4\end{array}\right]$

\section{Solución}

Teniendo presente los pasos para Descodificar o desencriptar un mensaje:

1. Lo primero que realizan es calcular el determinante de la matriz llave $\mathrm{A}_{3 \times 3}$, para verificar si es una matriz no singular (invertible), proceso que hacen por medio de la regla de Sarrus (método alternativo para el cálculo del determinante de una matriz $3 \times 3$ ).

$$
\operatorname{det}(A)=|A|=\left|\begin{array}{ccccc}
0 & 3 & 4 & 0 & 3 \\
1 & -4 & -5 & 1 & -4 \\
-1 & 3 & 4 & -1 & 3
\end{array}\right|=
$$

$$
\operatorname{det}(\mathrm{A})=|\mathrm{A}|=\begin{array}{ccccc}
0 & 3 & 4 & 0 & 37 \\
1 & -4 & -5 & 1 & -4=(0+15+12) \\
-1 & 3 & 4 & -1 & 3
\end{array}-(16+0+12)=27-28=-1 \text {, }
$$

Por lo tanto, concluyen que la matriz llave es no singular debido a $\operatorname{det}(\mathrm{A})=|\mathrm{A}| \neq 0$.

2. Se continúa con el cálculo de la matriz inversa $\left(\mathrm{A}^{-1}\right)$ de la matriz llave $\mathrm{A}_{3 \times 3}$, por medio del desarrollo de los determinantes y los cofactores de $\mathrm{A}_{3 \times 3}$. 


$$
\begin{aligned}
& A_{11}=(-1)^{2}\left|\begin{array}{cc}
-4 & -5 \\
3 & 4
\end{array}\right|=-1 \quad A_{12}=(-1)^{3}\left|\begin{array}{cc}
1 & -5 \\
-1 & 4
\end{array}\right|=1 \\
& A_{13}=(-1)^{4}\left|\begin{array}{cc}
1 & -4 \\
-1 & 3
\end{array}\right|=-1 \\
& A_{21}=(-1)^{3}\left|\begin{array}{ll}
3 & 4 \\
3 & 4
\end{array}\right|=0 \quad A_{22}=(-1)^{4}\left|\begin{array}{cc}
0 & 4 \\
-1 & 4
\end{array}\right|=4 \quad A_{23}=(-1)^{5}\left|\begin{array}{cc}
0 & 3 \\
-1 & 3
\end{array}\right|=-3 \\
& A_{31}=(-1)^{4}\left|\begin{array}{cc}
3 & 4 \\
-4 & -5
\end{array}\right|=1 \quad A_{32}=(-1)^{5}\left|\begin{array}{cc}
0 & 4 \\
1 & -5
\end{array}\right|=4 \quad A_{33}=(-1)^{6}\left|\begin{array}{cc}
0 & 3 \\
1 & -4
\end{array}\right|=-3 \\
& B=\left[\begin{array}{ccc}
-1 & 1 & -1 \\
0 & 4 & -3 \\
1 & 4 & -3
\end{array}\right]
\end{aligned}
$$

Donde B es la Matriz de cofactores de A

La matriz adjunta se definió como: $\operatorname{adj} \mathrm{A}=\mathrm{B}^{\mathrm{T}}$

$$
\mathrm{B}^{\mathrm{T}}=\left[\begin{array}{ccc}
-1 & 0 & 1 \\
1 & 4 & 4 \\
-1 & -3 & -3
\end{array}\right]=\operatorname{adj} \mathrm{A}
$$

entonces la matriz inversa de la matriz llave es:

$\mathbf{A}^{-1}=\frac{\mathbf{1}}{\operatorname{det}(\mathbf{A})} \operatorname{adj} \mathbf{A} ; \mathbf{A}^{-1}=\frac{\mathbf{1}}{-1}\left[\begin{array}{ccc}-1 & 0 & 1 \\ 1 & 4 & 4 \\ -1 & -3 & -3\end{array}\right] \mathbf{A}^{-1}=-\mathbf{1}\left[\begin{array}{ccc}-1 & 0 & 1 \\ 1 & 4 & 4 \\ -1 & -3 & -3\end{array}\right]$

Se aplica la multiplicación de un escalar por una matriz, para obtener:

$$
\mathbf{A}^{-\mathbf{1}}=\left[\begin{array}{ccc}
1 & 0 & -1 \\
-1 & -4 & -4 \\
1 & 3 & 3
\end{array}\right]
$$

Donde $\mathrm{A}^{-1}$ es la Matriz inversa de $\mathrm{A}$

3. Se procede a realizar el producto entre la matriz inversa de $\mathrm{A}_{3 \times 3}$ y la matriz codificada

$$
\left[\begin{array}{ccc}
-10 & 74 & 104 \\
-5 & 6 & 11 \\
-2 & 64 & 87 \\
16 & -7 & -4
\end{array}\right]\left[\begin{array}{ccc}
1 & 0 & -1 \\
-1 & -4 & -4 \\
1 & 3 & 3
\end{array}\right]=\left[\begin{array}{ccc}
20 & 16 & 26 \\
0 & 9 & 14 \\
21 & 5 & 7 \\
19 & 16 & 0
\end{array}\right]
$$

4. Para sustituir el carácter correspondiente a cada número de la matriz mensaje, se observa el código definido anteriormente, (ver Tabla I).

\begin{tabular}{|l|l|l|l|l|l|l|l|l|l|l|l|}
\hline 20 & 16 & 26 & 0 & 9 & 14 & 21 & 5 & 7 & 19 & 16 & 0 \\
\hline S & O & Y & $*$ & I & N & T & E & G & R & O & $*$ \\
\hline
\end{tabular}

La clase continua, para proponer que otro grupo (6) realice la codificación del mensaje SOY INTEGRO, el cual explica la codificación del mensaje de la siguiente manera:

1. El mensaje para codificar es SOY INTEGRO y como la matriz llave es del orden de $3 \times 3, A_{3 \times 3}$, entonces se tiene que agrupar de a tres letras o caracteres, (ver Codificar o encriptar el mensaje) como existe un espacio entre las palabras SOY e INTEGRO se debe escribir un * como se definió en el código Hill y además con las últimas dos letras se debe completar de a tres, por lo tanto, se tiene:

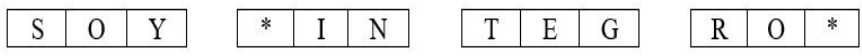

2. Continúan asignando el número que le corresponde a la letra o carácter según el código definido y se prosigue a formar por filas la matriz mensaje. 


\begin{tabular}{|c|c|c|}
\hline $\mathrm{S}$ & $\mathrm{O}$ & $\mathrm{Y}$ \\
\hline 20 & 16 & 26 \\
\hline
\end{tabular}$\quad$\begin{tabular}{|c|c|c|}
\hline$*$ & $\mathrm{I}$ & $\mathrm{N}$ \\
\hline 0 & 9 & 14 \\
\hline
\end{tabular}$\quad$\begin{tabular}{|c|c|c|c|}
\hline $\mathrm{T}$ & $\mathrm{E}$ & $\mathrm{G}$ \\
\hline 21 & 5 & 7 \\
\hline
\end{tabular}$\quad$\begin{tabular}{|c|c|c|c|}
\hline $\mathrm{R}$ & $\mathrm{O}$ & $*$ \\
\hline 19 & 6 & 0 \\
\hline
\end{tabular}

Por lo tanto, la matriz mensaje es:

$M=\left[\begin{array}{ccc}20 & 16 & 26 \\ 0 & 9 & 14 \\ 21 & 5 & 7 \\ 19 & 16 & 0\end{array}\right], M_{4 \times 3}$

3. Aplican el producto entre la matriz mensaje $\mathrm{M}_{4 \times 3}$ y la matriz llave $\mathrm{A}_{3 \times 3}$ para obtener la matriz codificada, donde va a estar el mensaje de Facebook en forma oculta.

$\left[\begin{array}{ccc}20 & 16 & 26 \\ 0 & 9 & 14 \\ 21 & 5 & 7 \\ 19 & 16 & 0\end{array}\right]\left[\begin{array}{ccc}0 & 3 & 4 \\ 1 & -4 & -5 \\ -1 & 3 & 4\end{array}\right]=\left[\begin{array}{ccc}-10 & 74 & 104 \\ -5 & 6 & 11 \\ -2 & 64 & 87 \\ 16 & -7 & -4\end{array}\right]$

Se termina el proceso de codificación del mensaje SOY INTEGRO, de forma correcta, manifestando que la única operación matricial que se tiene que realizar es la multiplicación de matrices.

\section{Resultados y análisis de la Implementación AEI}

Es importante mencionar, que al principio del desarrollo de la AEI, un número pequeño (3) estudiantes, presentaron resistencia al saber que el curso iban a presentarse cambios a la hora del desarrollo de los contenidos y la forma de evaluar, es decir, algo distinto a la educación tradicional como estaban a costumbrados, lo que implicó la intervención del docente y de otros estudiantes del curso a recordarles la metodología y las bondades de esta nueva forma de enfrentar el curso de Álgebra Lineal, a lo que se aclararon algunas dudas y "temores", a medida que se fueron realizando las actividades y el desarrollo del curso, fueron cambiando de actitud y presentaron un disposición positiva.

Para el análisis de la implementación de la primera Actividad de Estudio e Investigación (AEI1), se organiza la información en forma detallada en la tabla III , permitiendo evidenciar clases por clase la relación de las preguntas derivadas $\mathrm{Q}_{\mathrm{i}}$, las praxeologías $\mathrm{u}$ organizaciones matemáticas $(\mathrm{OM})$ encontradas y vinculadas en la construcción de cada una de ellas, siempre con el objetivo de dar respuesta a la pregunta generatriz $\mathrm{Q}_{0} \mathrm{y}$ a las preguntas derivadas en el desarrollo del estudio.

\section{Praxeologías construidas}

A continuación, se registran de forma resumida las praxeologías estudiadas en desarrollo de la AEI: OMP1: Definición de un matriz (DM); OMP2: Organización de las entradas (datos) en una matriz (OE); OMP3: Orden de una matriz y clases de matrices (OM y CM); OMP4: Operaciones con matrices y sus propiedades (OPM); OMP5: Operaciones elementales sobre las filas (renglones) de una matriz (OEFM); OMP6: Definición de matriz escalonada y escalonada reducida (DER); OMP7: Determinante de una matriz y sus propiedades (DETM); OMP8: Definición de matriz inversa y sus propiedades (DMI); OMP9: Cálculo de la matriz inversa por diferentes métodos (CMI); OMP10: Transformaciones lineales de matrices (TL).

OMP11: Sistemas de ecuaciones lineales de forma matricial $\mathrm{Ax}=\mathrm{b}$ y sistemas homogéneos $\mathrm{Ax}=0$ (SEL); OMP12: Método gráfico y reducción $\mathrm{o}$ eliminación gaussiana $\mathrm{y}$ Gauss-Jordán $\mathrm{Ax}=\mathrm{b}$. (EG y EGJ); OMP13: Matrices equivalentes y matriz de rotación (ME y MR); OMP14: Naturaleza de las soluciones de un sistema lineal (NSSL); OPM15: Regla de Cramer (RC); OPM16: Método de resolución por la matriz inversa $\mathrm{x}=\mathrm{A}^{-}$ ${ }^{1}$ b (SSMI); OPM17: Rango de una matriz (RM); OMP18: Definición de vectores, operaciones y sus propiedades (DOV); OMP19: Conjunto y espacio generador (CEG); OMP20: Sistema numéricos (decimal, binario, hexadecimal) (SN); OMP21: Matrices binarias (MB); OMP22: Aplicaciones de la codificación y simulación (ACS). 
Algunas de las anteriores praxeologías estudiadas, estaban contempladas de forma previa en el Marco Praxeológico de Referencia (MPR) en el diseño de la AEI y otras obedecen al surgimiento o la reconstrucción de estas, a medida que se iba implementado la AEI.

En la Tabla III se relacionan las praxeologías estudiadas por los estudiantes y su correspondencia con las preguntas derivadas Qi, las cuales se desarrollaron en cada clase.

Tabla III. Praxeologías estudiadas por los estudiantes y su correspondencia con las preguntas derivadas Qi

\begin{tabular}{|c|c|c|c|c|c|c|c|c|c|c|c|c|c|c|c|c|c|c|c|c|c|c|}
\hline$A E I$ & \multicolumn{22}{|c|}{ Praxeologías } \\
\hline $\begin{array}{l}\text { Preguntas } \\
\text { derivadas }\end{array}$ & DM & $\mathrm{OE}$ & $\begin{array}{c}\mathrm{OM} \\
\mathrm{y} \\
\mathrm{CM}\end{array}$ & OPM & OFM & DER & DETM & DMI & $\mathrm{CMI}$ & $\mathrm{TL}$ & SEL & $\begin{array}{l}\text { EG y } \\
\text { EGJ }\end{array}$ & $\begin{array}{c}\mathrm{ME} \\
\mathrm{y} \\
\mathrm{MR}\end{array}$ & NSSL & $\mathrm{RC}$ & SSMI & RM & DOV & $\mathrm{CEG}$ & SN & MB & $\mathrm{ACS}$ \\
\hline $\mathrm{Q}_{1}$ & $\checkmark$ & $\checkmark$ & & & & & & & & & & & & & & & & & & & & \\
\hline $\mathrm{Q}_{2}$ & $\checkmark$ & $\checkmark$ & & & & & & & & & & & & & & & & & & & & \\
\hline $\mathrm{Q}_{3}$ & $\checkmark$ & $\checkmark$ & $\checkmark$ & & & & & & & & & & & & & & & & & & & \\
\hline $\mathrm{Q}_{4}$ & & & & $\checkmark$ & $\checkmark$ & $\checkmark$ & $\checkmark$ & $\checkmark$ & $\checkmark$ & & & & & & & & & & & & & \\
\hline $\mathrm{Q}_{5}$ & & & & & & & $\checkmark$ & $\checkmark$ & $\checkmark$ & & & & & & & & & $\checkmark$ & & & & \\
\hline $\mathrm{Q}_{6}$ & & & & & & & $\checkmark$ & $\checkmark$ & $\checkmark$ & $\checkmark$ & $\checkmark$ & $\checkmark$ & $\checkmark$ & $\checkmark$ & $\checkmark$ & $\checkmark$ & $\checkmark$ & $\checkmark$ & $\checkmark$ & $\checkmark$ & $\checkmark$ & $\checkmark$ \\
\hline $\mathrm{Q}_{7}$ & & & & & & & & & & & & & & & & & & $\checkmark$ & $\checkmark$ & $\checkmark$ & $\checkmark$ & $\checkmark$ \\
\hline $\mathrm{Q}_{8}$ & & & & & & & & & & & & & & & & & & & $\checkmark$ & $\checkmark$ & $\checkmark$ & $\checkmark$ \\
\hline
\end{tabular}

Desde el inicio de la AEI, cada grupo de estudio estuvo en función de darle respuesta a la pregunta generatriz Q0 ¿Cómo se codifican o se encriptan mensajes?, donde se evidenció en el estudio y la investigación por parte de los estudiantes, en saber en un primer momento, definiciones y conceptos lo que dio lugar al surgimiento de preguntas derivadas ¿Qué es codificar o encriptar?, permitiendo determinar que existen diversas forma de codificación y de encriptar una información, donde el proceso de ocultar o cifrar un mensaje viene desde tiempos remotos utilizados por varias civilizaciones humanas en diferentes parte del mundo, por lo tanto, por la diversidad en la forma de codificar o encriptar se tiene el surgimiento de las preguntas ¿Qué clase de códigos es posible utilizar? ¿Con cuáles datos o entradas se puede codificar?, en el estudio e investigación de las respuestas a dichas preguntas, permitió abordar diferentes aspectos relacionados con varios códigos utilizados y las diferentes formas con se ha desarrollado la criptografía y cómo ha evolucionado a través del tiempo.
Por otro lado, el surgimiento de preguntas relacionadas con la codificación y con el objetivo de estudiar posibles OM del Álgebra Lineal se tiene ¿Qué codificación es posible realizar? ¿Qué condiciones y características se debe cumplir para codificar mensajes?, permitieron construir y reconstruir OMP relativas al algebra matricial, tanto en aspectos conceptuales, como procedimentales.

\section{Discusión}

Este estudio se une a otros ya desarrollados en el marco de la Teoría Antropológica de lo Didáctico (Chevallard, 1999; Bosch, García, Gascón, \& Ruíz 2006; Bosch, \& Gascón, 2009). García, Barquero, Florensa, \& Bosch, 2019), especialmente en elámbito del Algebra lineal (Costa, Rossignoli, Sorichetti \& Vampa, 2018; Trigueros, 2019; Álvarez-Macea, \& Costa, 2019; Alurralde, \& Ibarra, 2021) y afines (Costa, Arlego \& Otero, 2014). En este sentido se destaca la aplicación de una Actividad de Estudio e Investigación (AEI) que involucró a los estudiantes en un nuevo tipo de trabajo en el aula de matemáticas (Corica, \& Marin, 2014), para contribuir al propósito 
didáctico de matematizar las matemáticas, como herramienta que permite articular y dar sentido a la matemática que se enseña (Bernal, 2019), mediante la generación de cuestiones problemáticas cuyas respuestas desencadenarán organizaciones matemáticas, que constituyen la razón de ser del nuevo conocimiento a aprender. Por ello, se partió de una pedagogía no monumentalista (Gonzales, Papuico \& Cabrera, 2018) para ser aplicada en la enseñanza del Álgebra Lineal (Costa, Rossignoli, Sorichetti \& Vampa, 2018; Álvarez-Macea \& Costa, 2019), en un tema como es la codificación de mensajes (Velázquez-Rodríguez, 2014) con gran aplicabilidad en la ingeniería (Barría, Toledo \& Montenegro, 2018).

\section{Conclusiones}

En el marco de la Teoría Antropológica de lo Didáctico, específicamente con el dispositivo didáctico de la Actividad de Estudio e Investigación (AEI) relacionada con la codificación de mensajes utilizando organizaciones matemáticas y praxeologías del Álgebra Lineal, basados en un Marco Praxeológico de Referencia, se pudo desarrollar la AEI con el surgimiento de varias formas de codificar y decodificar mensajes a la medida que se iba estudiando e investigando para buscar posibles respuestas a la preguntas generatriz y sus derivadas. Con la implementación y análisis de una AEI por primera vez en un curso de Álgebra Lineal en carreras de Ingeniería, se evidenció una nueva forma en la enseñanza de Organizaciones Matemáticas (OM) diferente al modelo tradicional que habitualmente se lleva a cabo en las facultades de ingeniería.

Realizar un análisis praxeológico e instaurar los constructos teóricos de la Pedagogía de la Investigación y cuestionamiento del mundo, abre las posibilidades de repensar la enseñanza de las matemáticas o ciencias básicas en el contexto de la formación de los futuros ingenieros en sus áreas disciplinares, más reales. Por eso, al desarrollar la AEI relacionada con la codificación de mensajes, se evidencia que llevar este dispositivo didáctico al aula de clase permite minimizar en gran parte de la monumentalización del saber, tal como se concibe dentro de la TAD.

Es importante precisar que implementar nuevas alternativas pedagógicas y didácticas en un curso de Álgebra Lineal en la formación de ingenieros de diferentes disciplinas tiene sus resistencia por parte de los estudiantes, debido a la costumbre de estar recibiendo clases con una educación tradicional, por lo tanto, la experiencia llevada a cabo abre el abanico de realizar diferentes AEI en cursos de matemáticas donde la búsqueda de respuestas a una pregunta generatriz permite el estudio y el surgimiento de forma natural a diferentes contenidos, praxeologías y dialécticas específicas.

\section{Referencias}

Alurralde, F., \& Ibarra, L. (2021). El uso de las letras en álgebra: Análisis de una evaluación de estudiantes de primer año de ingeniería. Revista de Educación Matemática, 22, 1-10

Álvarez-Macea, F., \& Costa, V.A. (2019). Enseñanza del Algebra Lineal en carreras de ingeniería: un análisis del proceso de la modelización matemática en elmarco de la TeoríaAntropológica de lo Didáctico. Eco Matemático, 10(2), 65-78. https://doi.org/10.22463/17948231.2594

Barría, C. D., Toledo, Á. A., \& Montenegro, D. (2018). Aplicaciones del álgebra matricial en los cifrados por transposición. $I+T+$ C-Investigación, Tecnología y Ciencia, 1(12), 48-55

Bernal, A. (2019). Actividad de estudio e investigación para la enseñanza de nociones de funciones. Revista Virtu@Imente, 6(1), 27-47. https://doi.org/10.21158/2357514x. v6.n1.2018.2099

Bosch, M., \& Gascón, J. (2009). Aportaciones de 
la Teoría Antropológica de lo Didáctico a la formación del profesorado de matemáticas de secundaria. In Investigación en educación matemática XIII (pp. 89-114). Sociedad Española de Investigación en Educación Matemática, SEIEM

Bosch, M., García, F. J, Gascón, J., \& Ruiz, L. (2006). La modelización matemática y el problema de la articulación de la matemática escolar. Una propuesta desde la teoría antropológica de lo didáctico. Educación matemática, 18(2), 37-74

Chevallard, Y. (1985). La transposition didactique: du savoir savant au savoir enseigné. Paris, Francia: La Pensée Sauvage

Chevallard, Y. (1999). El análisis de las prácticas docentes en la teoría antropológica de lo didáctico. Recherches en Didactique des Mathématiques, 19(2), 221-266

Chevallard Y. (2001). Aspectos problemáticos de la formación docente, XVI Jornadas del Seminario Interuniversitario de Investigación en Didáctica de las Matemáticas, Huesca. http://yves. chevallard.free.fr

Chevallard, Y. (2004). Vers une didactique de la codisciplinarité. Notes sur une nouvelle épistémologie scolaire. Journées de didactique comparée, Lyon. France. http://yves.chevallard. free.fr/spip/spip/article.php3?id_article $=455$

Chevallard, Y. (2005). La place des mathématiques vivantes dans l'éducation secondaire : transposition didactique et nouvelle épistémologie scolaire. In C. Ducourtioux, P. L. Hennequin, (Éds.), La place des mathématiques vivantes dans l'enseignement secondaire (Vol. 168) (pp. 239-263). France: APMEP

Chevallard, Y. (2007). Passé et présent de la théorie anthropologique du didactique. In L. Ruiz,
A. Estepa, F. J. García, Sociedad, escuela y matemáticas. Aportaciones de la Teoría Antropológica de lo Didáctico (pp. 705-746). Universidad de Jaén, Servicio de Publicaciones

Chevallard, Y. (2009) Didáctica Fundamental: Foro de cuestiones. http://yves.chevallard.free.fr

Chevallard, Y. (2011) Improvisaciones cruzadas sobre lo didáctico, lo antropológico yel oficio de investigador en TAD. http://yves.chevallard. free.fr

Chevallard, Y. (2012). Théorie Anthropologique du Didactique \& Ingénierie Didactique du Développement. Journal du seminaire TAD/ IDD. http://www.aixmrs. iufm. fr/formations/ filieres/mat/data/fdf/2011-2012/journal-tadidd-2011-2012-7.pdf

Corica, A., Otero, M. (2012). Estudio sobre las praxeologías que se proponen estudiar en un Curso Universitario de Cálculo. Bolema. 26(42B), 459-482

Corica, A R., \& Marin, E. A. (2014). Actividad de estudio e investigación para la enseñanza de nociones de geometría. Números. Revista de Didáctica de las Matemáticas, 85, 91-114

Costa, V. A., Arlego, M. J. F., \& Otero, R. (2014). Enseñanza del Cálculo Vectorial en la Universidad: propuesta de Recorridos de Estudio e Investigación. Revista de formación e innovación educativa universitaria, 7(1), 20-40

Costa, V., Rossignoli, R., Sorichetti, C. \& Vampa, V. (2018). Algebra Lineal con Aplicaciones Parte I. Universidad Nacional de La Plata, La Plata: EDULP

García, F. J., Barquero, B., Florensa, I., \& Bosch, M. (2019). Diseño de tareas en el marco de la Teoría Antropológica de lo Didáctico. Avances 
de Investigación en Educación Matemática, 15, 75-94. DOI:10.35763/aiem.v0i15.267

Gonzales, C., Papuico, V., Cabrera, M. (2018). Modelización matemática en la formación de estudiantes de medicina. En L. Sema, D. Páges (Eds.), Acta Latinoamericana de Matemática Educativa (pp. 1344-1351). México, DF: Comité Latinoamericano de Matemática Educativa

Hernández, R., Fernández, C., \& Baptista, P. (2014). Metodología de la Investigación. México: McGraw-Hill

Rekalde, I., Vizcarra, M. T., \& Macazaga, A. M. (2014). La observación como estrategia de investiga-ción para construir contextos de aprendizaje y fomentar procesos participativos. Educación XX1, 17(1), 201-220. 10.5944/ educxx1.17.1.1074

Trigueros, M. (2019). Diálogo entre las teorías APOE y TAD Dialogue between APOE and ATD theories. Educ. Matem. Pesq., São Paulo, 21(5), 030-043. http://dx.doi.org/10.23925/19833156.2019v21i5p30-43

Velázquez-Rodríguez, C. E. (2014). HuffmanEncoder: Una aplicación informática para codificar mensajes por el método de Huffman. Ciencia \& Futuro, 4(3), 33-50 